\title{
Características de los programas de prevención primaria para el control del VIH en la región del suroeste antioqueño, 2014
}

\author{
Characteristics of the Primary Prevention Programs to Control HIV in the South-Western Antioquia
} Province, 2014

Características dos programas de prevenção primária para o controle da HIV na região do sul-oeste antioquenho, 2014

\author{
Mónica María Lopera Medina a \\ Universidad de Antioquia, Colombia \\ monica.lopera@udea.edu.co \\ ORCID: http://orcid.org/000-0003-0144-057X \\ Tatiana Betancur Montoya \\ Universidad de Antioquia, Colombia \\ ORCID: http://orcid.org/0000-0002-3973-6897
}

DOI: https://doi.org/10.11144/Javeriana.rgps17-35.cppp Redalyc: http://www.redalyc.org/articulo.oa?id=54557477006

\section{Resumen:}

Introducción: la prevención primaria (PP) del VIH/Sida es una obligación estatal para el control de la epidemia y el cumplimiento de los objetivos sanitarios, pero existe escasa evidencia sobre su funcionamiento. Objetivo: identificar algunas características de contexto, estructura, proceso y resultados de los programas de PP del VIH/Sida en el suroeste antioqueño. Método: estudio transversal, con análisis estadístico descriptivo, utilizando fuentes epidemiológicas y sociodemográficas ofi ciales y encuestas a 21/23 coordinadores de programa. Resultados: la incidencia, prevalencia y mortalidad del VIH/Sida fue de 10 casos por cada 100.000 habitantes, 73 casos por cada 100.000 habitantes y 22 casos por cada 100.000 habitantes. La disponibilidad de servicios fue limitada. La densidad profesional fue de 3 médicos/enfermeras/10.000 habitantes, coberturas de $\mathrm{PP}<25 \%$, focalizadas en actividades informativas, dirigidas principalmente a la población general, indígena y materna. Los coordinadores percibieron logros no cuantificados relacionados con disminución de transmisión madre-hijo, mayor uso de condón, pero también limitaciones económicas, de intersectorialidad y estigma. Conclusión: pese al impacto epidemiológico y a factores de riesgo contextuales, la estructura, los procesos y los resultados de PP requieren mejoramiento.

Palabras clave: evaluación de programas y proyectos de salud, infecciones por VIH, síndrome de inmunodeficiencia adquirida, prevención primaria, política pública.

\section{Abstract:}

Introduction: Primary prevention (PP) of HIV/AIDS is a state obligation in the control of the epidemy and to comply with the public health objectives, though there is scarce evidence on how it operates. Objective: To identify some characteristics of the context, structure, process and results of the HIV/AIDS primary prevention programs in the south-western Antioquia Province. Methods: It was a cross-sectional study with descriptive statistical analysis making use of the official sociodemographic and epidemiologic sources as well as surveys to $21 / 23$ program coordinators. Results: The incidence, prevalence and mortality of the HIV/AIDS 10 cases per 100,000, 73 cases per 100,000 and 22 per 100,000 inhabitants. The health services were limited, the density of health professionals was 3 doctors/nurses/10,000 inhabitants and the PP coverage was $<25 \%$, focused in informative activities targeting primarily the general population, indigenous populations and mothers. The program coordinators perceived non-quantified achievements related to a decrease in the mother-to-child transmission, a greater use of condoms but also fi nancial restrictions concerning intersectoriality and stigma. Conclusion: Despite the epidemiologic impact and contextualized risk factors, the structure, processes and results of the Prevention Programs require upgrading.

Keywords: program evaluation and health projects, HIV infection, Acquired Immune Deficiency Syndrome, primary prevention, public policy.

\section{Resumo:}

Introdução: a prevenção primária (PP) da Sida/AIDS/HIV é uma obrigação estatal para o controle da epidemia e o cumprimento dos objetivos sanitários, mas existe escassa evidência sobre seu funcionamento. Objetivo: identificar algumas

\footnotetext{
a Autora de correspondencia: monica.lopera@udea.edu.co
} 
características de contexto, estrutura, processo e resultados dos programas de PP da Sida/AIDS/VIH no sul-oeste antioquenho. Método: estudo transversal, com análise estatística descritiva a utilizar fontes epidemiológicas e sócio-demográfi cas oficiais e inquéritos a $21 / 23$ coordenadores de programa. Resultados: a incidência, prevalência e mortalidade da Sida/ HIV foi de 10 por $100.000,73$ por 100.000 e 22 por 100.000 habitantes. A disponibilidade de serviços foi limitada. A densidade profissional foi de 3 médicos/enfermeiros/10.000 habitantes, coberturas de $\mathrm{PP}<25 \%$, focalizadas em atividades informativas, dirigidas principalmente à população geral, indígena e materna. Os coordenadores perceberam êxito não quanti ficado relacionado com diminuição de transmissão mãe-filho, maior uso de camisinha, mas também limitações econômicas, de intersetorialidade e estigmatização. Conclusão: embora o impacto epidemiológico e fatores de risco contextuais, a estrutura, os processos e os resultados de PP precisam de melhoramento.

Palavras-chave: avaliação de programas e projetos de saúde, infeçes por HIV, síndrome de imunodeficiência adquirida, prevenção primária, política pública.

\section{Introducción}

La prevención integral de enfermedades como el VIH se entiende como un contínuum entre las acciones de prevención primaria, secundaria y terciaria. La prevención primaria incluye todas aquellas acciones dirigidas a evitar la aparición de las enfermedades mediante el control de los factores de riesgo, predisponentes y condicionantes que le sean asociados $(1,2)$. Las acciones de prevención primaria constituyen uno de los objetivos sanitarios más relevantes en el control del VIH. Ello implica la correcta y oportuna identificación de los diferentes grupos poblacionales, con un criterio incluyente, la consideración de las características contextuales relacionadas con la cultura y con los elementos socioeconómicos que incluyen y determinan los comportamientos individuales ligados a las prácticas cotidianas y sanitarias, pero también todas aquellas características institucionales y funcionales que permiten la provisión de programas eficaces a los fines, metas y propósitos sanitarios esperados.

En el Sistema General de Seguridad Social en Salud (SGSSS) existe una gran cantidad de normas, guías, orientaciones y lineamientos para el control de la epidemia $(3,4,5,6,7,8$, 9). Sin embargo, el sistema de salud separa la gestión del riesgo individual, de responsabilidad de las empresas administradoras de planes de beneficios (EABP), de las actividades colectivas de prevención primaria, cuya responsabilidad de dirección recae en el Estado a través de las secretarías territoriales de salud.

Este modelo no ofrece una visión integral para el manejo de la enfermedad y presenta importantes limitaciones en su operación $(10,11,12,13)$. Como consecuencia de esta mirada fragmentada, el país no ha logrado la eficacia suficiente para controlar la epidemia y mejorar la calidad de vida de los pacientes. Así, desde 1985 hasta el 31 de diciembre del año 2013, se notificaron 92.379 casos de VIH/sida (14). En el 2013, la incidencia de VIH en hombres fue de 25,6 casos por cada 100.000 habitantes y en mujeres de 9 casos por cada 100.000 habitantes, con variaciones importantes por departamentos. Los datos más recientes de país con relación a la transmisión maternoinfantil son de 2011, año en el que se registró un porcentaje de transmisión del 4,6\%. Ese mismo año, las coberturas de tamizaje para VIH en gestantes fueron de 69\% (15). Según datos oficiales, la epidemia en Colombia es concentrada, pero su prevalencia varía de acuerdo con el grupo poblacional. En estudios realizados en diferentes ciudades del país entre el 2002 y el 2012 se encontraron las siguientes prevalencias: en hombres que tienen sexo con hombres ( $\mathrm{HSH}$ ) 5,6 y 24\%, en mujeres transgénero entre 10 y $17 \%$, en trabajadoras sexuales entre 0,6 y 3,3\%, en usuarios de drogas intravenosas de 0,61 y $1,9 \%$, en jóvenes de $0,59 \%$ y en población privada de la libertad entre 0,5 y $0,6 \%$ (15).

Entre 1991 y el 2007, la tasa de mortalidad exhibió un patrón creciente que varió de 0,75 casos por cada 100.000 habitantes a 5,67 casos por cada 100.000 habitantes. Entre el 2008 y el 2012 se produjo una leve disminución; así, en el 2012 la tasa de mortalidad reportada fue de 4,76 casos por cada 100.000 habitantes, siendo para hombres de 7,3 y en mujeres de 2,2 casos por cada 100.000 habitantes (15). En el 2011, se calculó una carga de enfermedad por VIH de 1.013.444 AVPP (años de vida potencialmente perdidos) por cada 100.000 habitantes (16). 
En Antioquia, en el 2015, la tasa de incidencia fue de 26,9 casos por cada 100.000 habitantes y la región de suroeste alcanzó una tasa de 14,3 casos por cada 100.000 habitantes. Excluyendo la región del valle de Aburrá, cuyos datos pueden estar distorsionados por ser sitio de referencia de las demás regiones, el suroeste está dentro de las tres regiones con mayor prevalencia en el departamento de Antioquia, solo superada por regiones como Magdalena medio, Occidente y Bajo Cauca (17).

El VIH es una prioridad en la agenda nacional e internacional. Colombia se unió a la política de Objetivos de Desarrollo del Milenio (ODM) y Objetivos de Desarrollo Sostenible (ODS) del 2000 y del 2015, respectivamente. Uno de los ODS es garantizar una vida sana y promover el bienestar para todos en todas las edades. Para el VIH, específicamente, se plantea terminar en el 2030 la epidemia del sida. Para ello, propone como estrategia la información, la educación y la integración de la salud reproductiva en las estrategias y programas nacionales (18).

En el reciente Plan Decenal de Salud Pública, así como en guías, normas y planes anteriores, se describe como fundamental la incorporación de estrategias que permitan la prevención del VIH, la reducción de condiciones de vulnerabilidad, la prevención de la transmisión, tanto sexual como de la transmisión maternoinfantil, así como la prevención del estigma. Para ello, estas políticas y lineamientos proponen la coordinación sectorial, transectorial y comunitaria para la gestión de políticas y ponen énfasis especial en la atribución de responsabilidades concretas a las EPS y en la participación de ONG y otros actores sociales.

En el Decreto 1543 de 1997 se destacan los elementos de prevención como aquellos encaminados a mejorar los procesos de educación e información, la incorporación de servicios sociales y de salud y la promoción de la tolerancia social, basada en el respeto de los derechos sexuales y reproductivos (4). Por su parte, el modelo de gestión programática formulado en el 2006 busca centrar sus acciones en la fase previa al proceso patológico, mediante la modificación de los macrodeterminantes de la transmisión, pero también de la implementación de acciones concretas de protección específica y su articulación con aquellas acciones realizadas por la red primaria de atención del usuario y el ámbito comunitario. Las intervenciones de protección específica buscaban mejorar el acceso a los preservativos, motivando la disposición masiva, promover los comportamientos protectores y reducir las prácticas de riesgo (5).

Desde un plano operativo, todos los lineamientos propuestos coinciden en el fomento de estrategias educativas para la prevención de la infección, incluidas aquellas que provengan de difusión de mensajes mediante medios masivos de comunicación, acciones de prevención de tipo comunitario para la detección temprana y canalización de los servicios de salud, información, educación y comunicación para la reducción del estigma y la discriminación hacia personas que viven con VIH, prevención de la transmisión maternoinfantil mediante el suministro de tratamiento a mujeres embarazadas y leche de fórmula a los recién nacidos, promoción de la asesoría y prueba voluntaria y el análisis de la información diagnóstica, terapéutica y operativa, prevención (y atención) de la coinfección $\operatorname{VIH} / \mathrm{TB}(6,9)$.

Con el fin de garantizar el acceso universal y equitativo a los servicios de salud, el Plan Decenal de Salud Pública ordena muy especialmente la inclusión de grupos vulnerables en virtud de sus orientaciones sexuales diversas, condiciones socioeconómicas adversas relacionadas con condición de calle, población privada de la libertad, usuarios de drogas intravenosas y jóvenes en contextos de vulnerabilidad (19). Por último, el Decreto 1543 plantea la obligatoriedad de las IPS y profesionales de brindar una atención integral a quienes viven con $\mathrm{VIH} /$ sida, de acuerdo con el nivel de atención y la complejidad (4).

En contravía de los lineamientos que normatizan las actividades de salud pública, las intervenciones colectivas y las directrices para procurar el derecho a la salud, mediante la unificación del Plan Obligatorio de Salud ordenada por la sentencia T-760 y la Ley 1438 (7, 10, 19, 20, 21), la población tiene un acceso diferencial a las actividades individuales de prevención y de atención (prevención secundaria y terciaria), de acuerdo con sus condiciones de vida, su estatus de afiliación, su orientación sexual y su área de residencia (22). Sin embargo, se desconoce cómo se están realizando los programas de prevención primaria en los municipios que normativamente están a cargo del Estado. 
En el país existen muy pocas investigaciones que intenten determinar el desempeño de los programas de prevención primaria (23) y ninguna en la región del suroeste que analice estos elementos a la luz de los contextos municipales en los que se desarrollan. Esta región se encuentra entre las cuatro con mayor incidencia de VIH. Las investigaciones existentes se enfocan predominantemente en la percepción de la calidad de los servicios o condiciones de los pacientes diagnosticados con VIH y en los factores de riesgo relacionados con las poblaciones especiales (24), pero ninguna en nuestro conocimiento que intente determinar los atributos de los programas de prevención primaria, de acuerdo con los contextos municipales, los recursos capitales y los resultados alcanzados en términos de coberturas en las distintas poblaciones. Esta deficiencia en el conocimiento de los procesos sanitarios impide efectuar mecanismos de mejoramiento que permitan prevenir los nuevos casos, efectuar diagnósticos oportunos y por esta vía controlar la expansión de la epidemia y reducir los altos gastos de atención en salud que representa el VIH para el sistema (25).

Como un esfuerzo por acoger un modelo teórico integrativo, se han retomado elementos de varios enfoques: el modelo teórico político-económico (26), la perspectiva teórica del análisis de calidad de los servicios que propone Donabedian (27), algunos elementos de disponibilidad propuestos por la Organización Mundial de la Salud (OMS) y el componente de prevención primaria propuesto por Leavell y Clark, ajustado a los procesos básicos que amerita el manejo particular del VIH (28).

Desde la perspectiva político-económica acogida, se reconoce que la epidemia del VIH se sustenta en causas económicas, políticas, culturales y sociales, pero también desde la función estructural en la distribución de los recursos capitales con los que cuenta la sociedad, los cuales están dispuestos de manera desigual entre diferentes grupos sociales. Bajo esta perspectiva, el análisis de la estructura y el funcionamiento de los programas se entiende en el marco de las características contextuales y la distribución de los recursos. Desde este enfoque, las características contextuales constituyen elementos fundamentales para la comprensión del fenómeno del VIH.

Esta perspectiva también implica la necesidad de entender que el control del VIH obliga a instaurar procesos integrados y continuos entre la prevención primaria, secundaria y terciaria propuesta por Leavell y Clark. La primera, referida a los mecanismos que evitan la presentación de la enfermedad, la segunda entendida como aquellos que intentan mantener estándares de salud y evitar la progresión en los ya afectados, y la terciaria, como los que evitan la discapacidad y muerte en pacientes enfermos (22). Este estudio solo abordará la determinación de atributos de los procesos concernientes a la prevención primaria de carácter colectivo, que en el sistema son entendidas como aquellas actividades de salud pública a cargo de las entidades territoriales, en concertación con los otros actores sociales.

Para identificar las características propias de los programas, se retomó la perspectiva teórica del análisis de calidad de los servicios que propone Donabedian (27), que contiene tres elementos básicos: estructura, procesos y resultados. Para el análisis de la estructura, se acoge el concepto de disponibilidad sugerido por la OMS. Estos permiten identificar factores relacionados con las condiciones de capacidad instalada y las características del personal que opera en los programas para la prevención primaria del VIH en los contextos locales (29). En la categoría de procesos se incluyen la articulación interinstitucional para el ejercicio de planificación de los programas y el tipo de actividades de prevención primaria. Por último, como resultados del programa se entienden las coberturas logradas para cada una de las actividades estudiadas y las condiciones epidemiológicas (incidencia, prevalencia y mortalidad). Desde el marco conceptual acogido, los resultados surgen como consecuencia de la confluencia de los elementos estructurales y funcionales de los programas, bajo la influencia de los contextos sociodemográficos, políticos y económicos y no como efecto directo de la aplicación de los programas o actividades.

A partir de la identificación de los vacíos de conocimiento, la importancia del análisis de los programas en salud y la viabilidad política alcanzada, se propone identificar las características de los programas de prevención primaria para el control del VIH en la región del suroeste antioqueño, con miras a brindar elementos que permitan a los distintos actores del sistema implementar medidas para su mejoramiento. Este 
es el primer esfuerzo por entender las dinámicas de los programas de VIH en Antioquia y constituye una base para futuros estudios en el resto de las regiones del departamento.

\section{Método}

Estudio cuantitativo descriptivo y transversal. La información de cada municipio contenía datos de contexto, estructura, proceso y resultados de los programas. Los datos de contexto y resultados epidemiológicos se tomaron de fuentes primarias, que consistieron en información oficial captada para el total de municipios de la región del suroeste. Estos datos provienen de las bases de datos de mortalidad del DANE, la encuesta del Sisben, el Sistema de Información Sivigila y la Cuenta de Alto Costo (CAC). Los datos de estructura, procesos y resultados (referidos a las coberturas) se recolectaron a partir de una encuesta semiestructurada diseñada por el equipo de investigación, con base en la revisión de literatura y aplicada a los coordinadores del programa. Esta encuesta fue construida a partir de una amplia revisión bibliográfica y sustentada en el modelo conceptual integrado que se mencionó antes. La encuesta consta de 48 preguntas y se aplicó a 19 de los 23 coordinadores de promoción y prevención que tenían experiencia de al menos tres meses liderando el programa (unidad de observación) y que decidieron voluntariamente participar y firmar el consentimiento informado.

Las variables de contexto incluyeron el índice multidimensional de calidad de vida (IMCV), la categorización presupuestal, el tamaño del municipio y la tipología, la densidad poblacional y otras características sociodemográficas básicas como distribución por edad, tasa de analfabetismo, distribución poblacional por régimen de afiliación y características especiales (28). El IMCV es un índice calculado por Planeación Departamental de Antioquia, que oscila entre 0 y 100, siendo 100 el más alto nivel de calidad de vida. La categorización del municipio se determinó según la Ley 617 del 2000 (30). El tamaño del municipio asumió dos categorías: grupo 1 y grupo 2. El grupo 1 estuvo conformado por aquellos municipios constituidos por menos de 10.000 habitantes en la zona urbana y el grupo 2 por aquellos que tenían entre 10.000 y 32.000 habitantes. Los estándares de clasificación del municipio según el tamaño y el predominio urbano-rural fueron los propuestos por Posada. Otras características sociodemográficas básicas incluyeron el número de habitantes por grupo de edad, régimen de afiliación y poblaciones consideradas especiales por la vulnerabilidad relacionada con el VIH.

La estructura de los programas incluyó lo referente a la disponibilidad de la red de servicios y el tipo de personal encargado de las actividades de prevención primaria y su respectiva densidad profesional, así como los aspectos de coordinación intersectorial relacionados con la planificación de los programas.

En la categoría de procesos se incluyeron dos aspectos: la coordinación interinstitucional relacionada con la planificación de los programas y algunas actividades específicas para la prevención primaria, según estándares de cuidado y orientaciones normativas $(31,32)$. Para cada actividad de prevención primaria y secundaria, se calcularon las respectivas coberturas logradas, tanto para población general (de 25 a 65 años) como para población especial, utilizando para ello los denominadores de la base poblacional respectiva, obtenida en la base de datos nacional del Sisben. Estas coberturas se muestran agregadas para la región. En la categoría de resultados se incluyeron los indicadores básicos de prevalencia, incidencia y mortalidad por VIH, extraídos de fuentes secundarias provenientes de los reportes sobre los eventos de salud pública departamentales, calculados por la gobernación de Antioquia. Se calculó la tasa ajustada para los municipios de la región. También se indagó por los resultados sanitarios percibidos por los coordinadores.

\section{Análisis de la información}

Se realizó análisis univariado, utilizando estadísticos descriptivos con sus respectivas medidas de tendencia central. Para ello, se determinó la normalidad de la distribución de los datos con la prueba de KolmogorovSmirnov. Se determinaron proporciones, teniendo como base poblacional los datos oficiales del Sisben 
municipal. Los datos fueron analizados con ayuda de los programas estadísticos SPSSS versión 22 (SPSS, Chicago, IL) y ArcGIS versión 10. Los resultados se presentan en figuras, tablas y mapas.

El proyecto acogió las consideraciones éticas nacionales e internacionales $(33,34,35,36)$ y fue avalado por el Comité de Ética de Investigación de la Facultad Nacional de Salud Pública en su sesión 127 del 17 de septiembre del 2015.

\section{Resultados}

\section{Contexto de los municipios de la región}

Los 23 municipios del suroeste pertenecen a la categoría 6. La región presenta IMCV que varían entre 32,3 y 35,7 . El 70\% son municipios pequeños (población urbana menor a 10.000 habitantes), con similar proporción urbana y rural (40\% para cada una). La población total en la región del suroeste fue de 376.697 habitantes y varió entre 4000 a 46.000 habitantes en los diferentes municipios (mediana de 13.834 RI: 13.623). La mediana de la densidad poblacional fue de 76 habitantes por cada $\mathrm{km}^{2}$, con resultados variables entre los municipios. Esta osciló entre 17 y 349 para los extremos. Entre su población, se encontró una tasa de analfabetismo de 9,6\%. En la región predominó el régimen subsidiado, con el 60\%, y aún existía un $18 \%$ de población pobre no asegurada (PNA). Para el periodo de estudio, se reportaron en la región 42.640 desplazados $(0,1 \%)$ y 590 indígenas, con un porcentaje mínimo de 0,001\%. Las características contextuales de la región se presentan en la tabla 1. 
TABLA 1

Contexto de los municipios del suroeste antioqueño para el año 2014

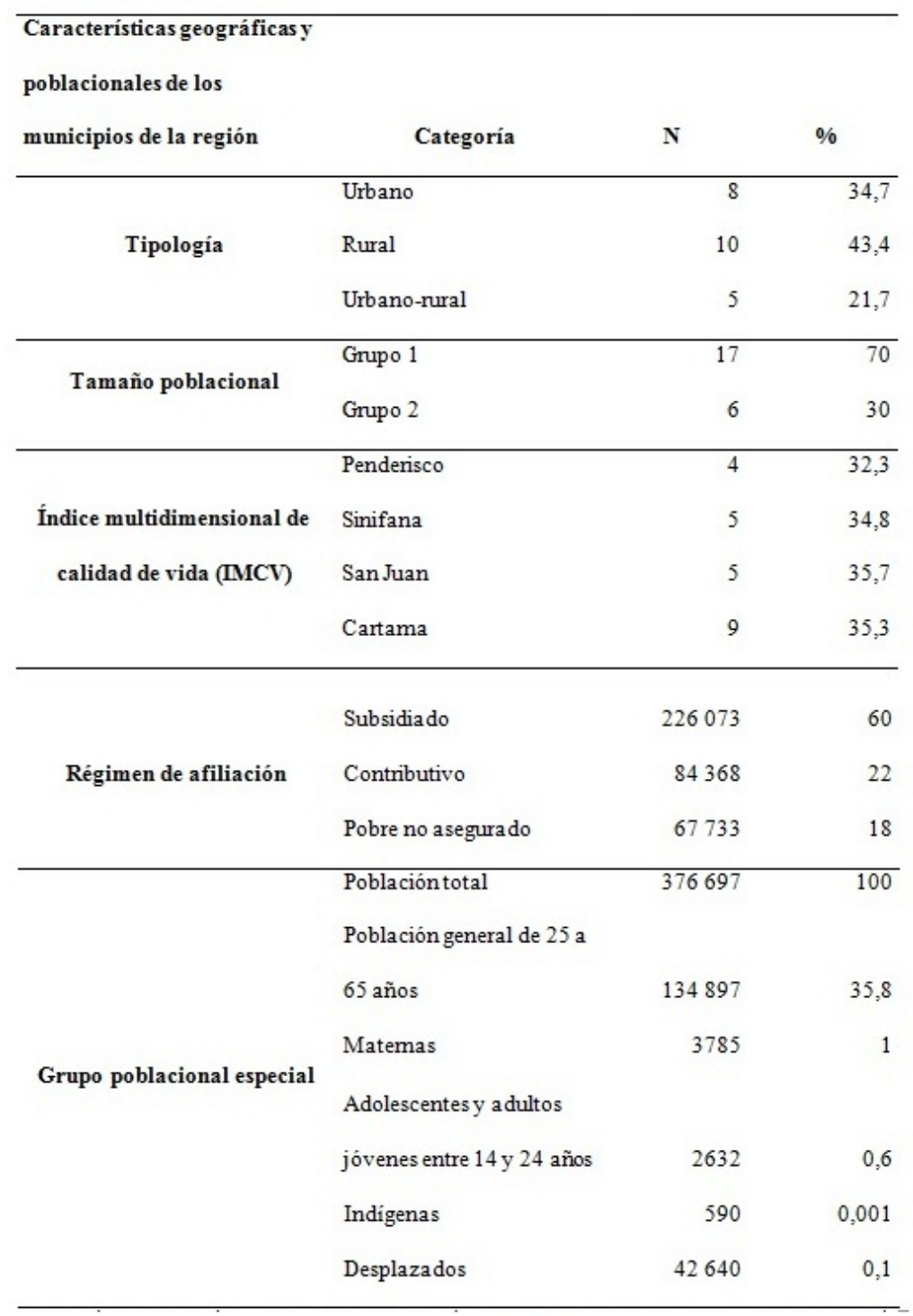

La población total de la región fue de 376.697 personas y osciló entre 4000 a 46.000, de acuerdo con los municipios de la región del suroeste. Fuente: elaboración propia, basada en los indicadores de salud de la Gobernación de Antioquia. 


\section{Estructura de los programas para el manejo del VIH}

\section{Disponibilidad}

Los programas para el control del VIH están incluidos en las actividades de promoción y prevención, coordinados y ejecutados predominantemente por funcionarios de la Empresa Social del Estado (ESE) (> $84 \%$ de los municipios). En cuanto a la red de servicios, todos los municipios contaban con una ESE de primer nivel, excepto un municipio responsable del segundo nivel de atención.

Con relación a la disponibilidad del talento humano en la región, se contaba con 127 médicos generales, 3 médicos especialistas, 22 enfermeros profesionales, 58 auxiliares de enfermería y 27 profesionales de otras áreas. La densidad profesional de la región fue de 3 médicos y enfermeros por 10.000 habitantes. El 18\% de los médicos generales y el 100\% de los especialistas trabajan tiempos parciales. El 68\% de los municipios de la región no contaba con grupos interdisciplinarios y el 11\% tenía grupos de apoyo para el manejo del VIH.

\section{Procesos}

\section{Coordinación interinstitucional}

La coordinación interinstitucional fue estudiada mediante la valoración de la integración de las actividades de prevención entre el ejecutor con las EPS y la autoridad sanitara. Entre el 47 y el 53\% de los coordinadores reconocieron el apoyo de las secretarías de salud municipal y departamental como satisfactoria y entre el 21 y el 26\% como baja. El 37\% de los municipios expresó coordinación con todas las EPS que operan en el territorio; en el 47\% de los casos la participación fue de algunas EPS y en el 5\% de los municipios, no hubo participación por parte de estas instituciones.

\section{Actividades de prevención primaria}

Los programas de prevención primaria incluyeron campañas para pruebas de VIH y tamización, charlas informativas, asesorías pretest y distribución de condones. Las actividades se realizaron en el $88 \%$ en la zona urbana y en el 71\% en la zona rural. El 5\% de los municipios no reportó actividades de prevención primaria.

Las actividades de prevención primaria estuvieron dirigidas principalmente a la población general. Las coberturas alcanzadas en esta población fueron inferiores a 1,8\%. Por falta de información en las bases de datos del Sisben no fue posible calcular las coberturas de actividades para la prevención del VIH en otras poblaciones especiales, como los $\mathrm{HSH}$ y los trabajadores sexuales.

Las charlas informativas y la tamización, dirigidas a la población general y los adolescentes y adultos jóvenes, se realizaron en el 37 y $32 \%$ de los municipios, respectivamente, y las coberturas oscilaron entre 0,5 y $7,1 \%$. Estas se enfocaron en la toma de medicamentos, estilos de vida, modos de transmisión, riesgos para los convivientes, recomendaciones alimentarias, autocuidado y asesorías sobre estilos de vida. En el caso de las maternas, la cuarta parte de los municipios reportó actividades educativas y la cobertura alcanzada fue de 13\%. Las campañas para las pruebas del VIH fueron realizadas entre el 16 y $37 \%$ de los municipios, con coberturas inferiores al 3\%. La mayor cobertura para las asesorías pretest se lograron para las maternas (24,6\%). La distribución de condones se realizó entre el 21 y el $42 \%$ de los municipios y las coberturas fueron inferiores a 3,5\%.

Ningún municipio reportó desarrollar pruebas de VIH para recién nacidos, hijos de madres infectadas. El 26,3\% de los municipios realizaban tratamiento y atención del VIH, así como el seguimiento de la adherencia 
a los pacientes con VIH. Las actividades de prevención primaria y las respectivas coberturas se muestran en la tabla 2.

TABLA 2

Actividades de prevención primaria para el control del VIH y cobertura de actividades entre la población

\begin{tabular}{|c|c|c|c|c|c|}
\hline \multirow{3}{*}{$\begin{array}{l}\text { Actinidades de prereación para } \\
\text { el vilH }\end{array}$} & \multirow{3}{*}{ Poblaciōn } & \multirow{2}{*}{\multicolumn{2}{|c|}{ Muaicipbs }} & \multirow{2}{*}{\multicolumn{2}{|c|}{$\begin{array}{l}\text { Cobertura } \\
\text { poblacional }\end{array}$}} \\
\hline & & & & & \\
\hline & & $\mathrm{x}$ & 96 & $\mathrm{~s}$ & 96 \\
\hline Charlas informativas $y$ & Poblation geneal & 7 & 36,8 & 1565 & 0.5 \\
\hline educaticas para preveación del & Adolescertes y ábilos jóvenes & 6 & 31,6 & 6310 & 7,1 \\
\hline VIH & Menerasa & 5 & 26,3 & 498 & 13,2 \\
\hline \multirow[t]{4}{*}{$S=16$} & HSH & 2 & 10,5 & 4 & SD \\
\hline & TS & 1 & 5,3 & 6 & SD \\
\hline & Nin̄os < 14 aี่os & 2 & 10,5 & 15 & 0,02 \\
\hline & Desplazados & 1 & 5,3 & 100 & 0.3 \\
\hline Campatiss para pruebas del & Poblasion gencel & $T$ & 36,8 & 164 & 0,06 \\
\hline VIH en grupes especificos & Adolescestes y abullos jóvenes & 3 & 15,8 & 177 & 0.2 \\
\hline$x-7$ & Matemas & 3 & 15,8 & 110 & 29 \\
\hline Asesoria pretest & Poblacisa general & 7 & 36,8 & 377 & 0,1 \\
\hline \multirow[t]{4}{*}{$N=13$} & Adolescentes y a aultos jorremes & 3 & 15,8 & 10 & 0,01 \\
\hline & Matenas & 12 & 63,2 & 933 & 24,6 \\
\hline & HSH & 2 & 10,5 & 2 & SD \\
\hline & Persouas en situación de calle & 1 & 5,3 & 1 & $\mathrm{SD}$ \\
\hline Asesoria postest & Poblackiu general & 6 & 31,6 & 431 & 0,15 \\
\hline \multirow[t]{3}{*}{$\mathrm{N}=8$} & Adolescentes y adultos jorremes & 1 & 5,3 & 6 & 0,01 \\
\hline & Matremas & 3 & 15,8 & 79 & 2,09 \\
\hline & $\mathrm{HSH}$ & 1 & 5,3 & 2 & SD \\
\hline Distribuçion de condones & Poblacisa general & 8 & 42,1 & 5252 & 1,8 \\
\hline \multirow[t]{4}{*}{$N=9$} & Adolescentes y sdultor jovienes & 4 & 21,1 & 3000 & 3,4 \\
\hline & TS & 2 & 10,5 & 1000 & SD \\
\hline & HSH & 2 & 10,5 & 100 & SD \\
\hline & Indigenas & 1 & 5,3 & 100 & 2,3 \\
\hline $\begin{array}{l}\text { Profilaris postexposicion } \\
\mathrm{N}=1\end{array}$ & Población genenal & $T$ & 3,3 & 1 & $\sigma$ \\
\hline $\begin{array}{l}\text { Accones para ecitar la } \\
\text { exchsión social para bs } \\
\text { persouas con VIH } \\
\mathrm{N}-3\end{array}$ & Poblacion geveral & 3 & 15,8 & 14 & $\sigma$ \\
\hline $\begin{array}{l}\text { Control prenatal para } \\
\text { enbaraxadas a quientes se } \\
\text { realiran prueba de VIH } \\
\mathrm{N}=4\end{array}$ & Matemas & 4 & 21,1 & 276 & 7,29 \\
\hline
\end{tabular}

$\mathrm{HSH}$ : hombres que tienen sexo con hombres.

TS: trabajadores sexuales.

Población general: población de 25 a 65 años.

Sin dato (SD): los municipios no tienen censo de algunas poblaciones especiales como las trabajadores sexuales y HSH.

Fuente: elaboración propia 


\section{Resultados en salud: incidencia, prevalencia y mortalidad}

Entre el 2007 y el 2014 en la región se reportaron 274 personas con VIH. Para el 2014, la prevalencia fue de 70 casos por cada 100.000 habitantes; la incidencia fue de 10 casos por cada 100.000 habitantes. En esos mismos años, el número de muertes fue de 89 personas (32\%), lo que reporta una tasa de mortalidad de 22 casos por cada 100.000 habitantes (figura 1).

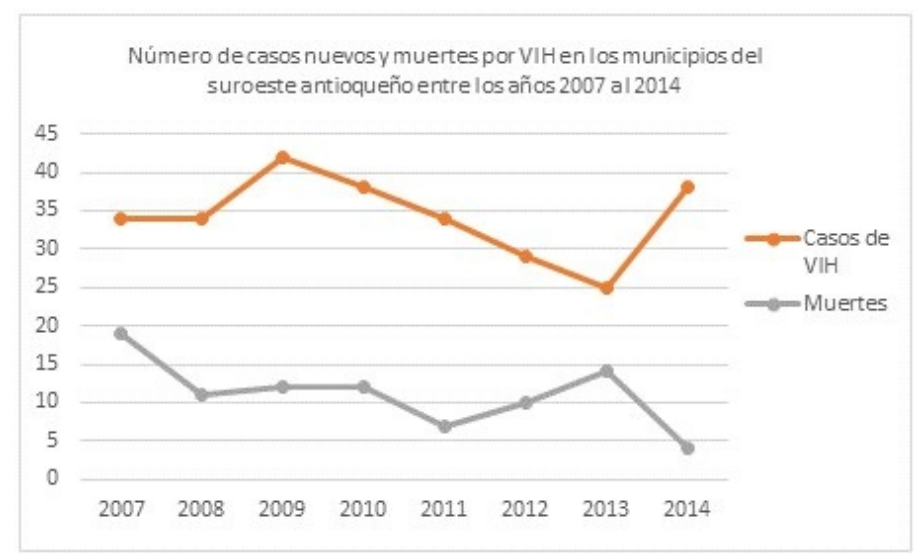

FIGURA 1

Número de casos nuevos y muertes por VIH en los municipios del suroeste antioqueño entre los años 2007 y 2014.

Fuente: elaboración propia.

El 64\% de los participantes diagnosticados con VIH pertenecían al régimen subsidiado, y el 36\% al régimen contributivo. No se reportaron pacientes con VIH en el grupo de los pobres no asegurados. Los municipios más afectados por la epidemia fueron Andes y Venecia, seguidos por Fredonia y Amagá. El mapa con la respectiva prevalencia e incidencia de VIH para la región se observa en la figura 2.

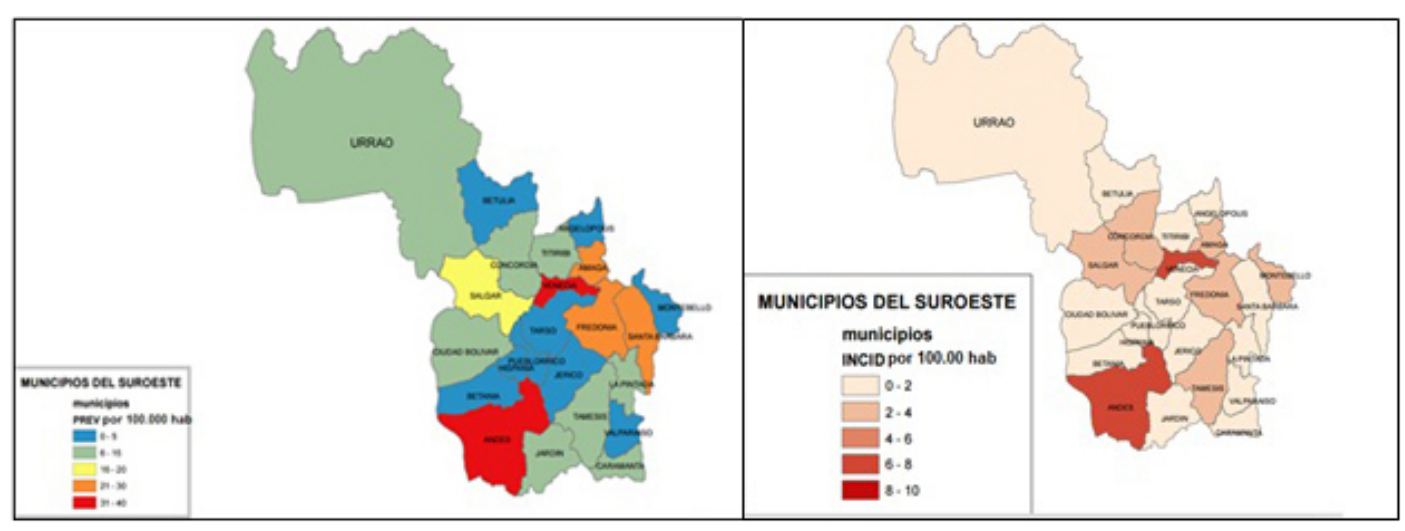

FIGURA 2

Prevalencia e incidencia en los municipios del suroeste antioqueño, 2014 Fuente: elaboración propia. 


\section{Resultados sanitarios percibidos por los coordinadores. Logros y retos}

Los resultados percibidos por los responsables del programa están relacionados con la reducción de la transmisión madre-hijo, la mayor utilización de condón, la disminución de los embarazos en adolescentes infectados y la disminución de la discapacidad y la mortalidad. Sin embargo, los municipios no contaban con cifras oficiales, puesto que este tipo de impacto no es monitorizado sistemáticamente.

A pesar de estos logros percibidos, los coordinadores plantearon que tienen retos relacionados con los mitos y prejuicios sobre el VIH, la superación de barreras de tipo político, religioso y geográfico afines con lugares apartados, así como barreras administrativas, limitada participación de otros actores y el deficiente presupuesto.

\section{Conclusiones}

Los municipios de la subregión tienen condiciones estructurales de pobreza potencialmente favorecedoras de la expansión de la epidemia: todos los municipios pertenecían a la categoría 6 , con índice de calidad de vida cercanos a 30 (por debajo del promedio departamental estimado en 41,5), tasas promedio de analfabetismo cercanas al 10\%, cifra por encima del promedio departamental, estimado en 4,7, y una alta población perteneciente al régimen subsidiado. El 43,4\% de la población es rural y dispersa. Las condiciones socioeconómicas desfavorables se han visto involucradas como factores determinantes de la epidemia (37).

A pesar de las condiciones anteriormente descritas, los municipios de la región contaban con una infraestructura sanitaria muy básica y una oferta de servicios de prevención predominantemente centralizada en las cabeceras municipales. Este estudio encontró una limitada disponibilidad del talento humano. La densidad profesional se calculó en tres médicos y enfermeras/10.000 habitantes. De acuerdo con los estándares de OMS (38), los territorios deben estar por encima de 50 por cada 10.000. En particular, se encontró que la región solo cuenta con tres especialistas que trabajaban a tiempo parcial (11). La participación de otros profesionales no médicos, que desempeñan un papel importante en la provisión de una atención integral, también es escasa. También se evidenció que no existen grupos interdisciplinarios para el manejo del VIH y los grupos de apoyo son escasos.

Rodríguez afirma que Colombia se ha caracterizado por tener un sistema de salud deficiente y muy pocos profesionales para la demanda de atención $(23,24)$. Por otro lado, Knobel et al. explican la importancia de personal suficiente y capacitado para el control de la epidemia (39).

Los programas de prevención primaria se realizaron en menos del $25 \%$ de los municipios y las coberturas fueron muy bajas. Estos programas estuvieron dirigidos predominantemente a la población general y a las maternas, pero dejan descubiertas a otras poblaciones especiales que ya han sido reconocidas con mayor vulnerabilidad para la infección con el VIH. Aunque las actividades se realizaron tanto en la zona urbana como en la rural, la frecuencia fue mayor en la primera, lo que pudiera estar afectando la equidad sanitaria (40).

Las bajas coberturas en actividades básicas de prevención primaria constituyen un factor de riesgo que favorece la presentación y atención tardía, cuando por lo general los pacientes tienen signos y síntomas compatibles con sida y otras complicaciones. Además de estas consecuencias clínicas, la falta de actividades preventivas facilita la expansión de la epidemia, el aumento del gasto en salud, tanto para los pacientes como para el sistema, como ya se ha evidenciado en otras investigaciones $(11,12)$.

Los datos epidemiológicos consultados permiten afirmar que los resultados en salud fueron poco satisfactorios. La prevalencia de VIH en la región fue de 73 casos por cada 100.000 habitantes. Esta cifra está por encima del promedio departamental y nacional que alcanzó 22 y 27 casos por cada 100.000 habitantes, respectivamente, en el año de estudio (41). Con relación a la incidencia y la 
mortalidad, los municipios de la región se ubicaron por debajo de las cifras departamentales y nacionales, pero desde el 2009 se observa una tendencia de aumento de la mortalidad (42). No es posible determinar si los datos de la situación epidemiológica obtenidos a partir de fuentes secundarias están relacionados con subdiagnósticos, subregistros o identificación de casos en otros municipios como Medellín. En Colombia, es común que existan discrepancias de información entre las diferentes fuentes de notificación y registro, aspecto del que no estuvo exenta la subregión estudiada.

De acuerdo con el Foro Latinoamericano y del Caribe del 2015, entre el 2010 y el 2014 solo se ha obtenido una reducción de 3\% en la incidencia de la enfermedad (43). Por la naturaleza de este estudio, las fuentes de información y la fragmentación de esta, los resultados de logro relacionados con la disminución de la incidencia en el suroeste se desconocen, y por lo tanto no se sabe si la epidemia se ha estabilizado o no.

Según datos obtenidos por algunos investigadores, en muchos países con alta mortalidad por VIH es usual que los sistema de salud sean débiles y es probable que puedan estar en crisis frente a la epidemia. También es frecuente que se cuente con escasos recursos para el VIH, que los programas sean desestimados o que los recursos sean invertidos en otros programas (44).

En este estudio se encontró que el $64 \%$ de las personas con VIH pertenecían al régimen subsidiado, lo que puede sugerir inequidades sociales y sanitarias. Estas inequidades han sido reportadas también por otros autores (45). Por sus condiciones socioeconómicas, la población del régimen subsidiado es una población vulnerable y carece de acceso oportuno y continuo al sistema de salud (42). La literatura nacional también permite concluir que esta población tiene cifras de prevalencia, incidencia y complicaciones mayores que las pertenecientes a otros regímenes (13).

A pesar de que para la fecha del estudio la región tenía un $18 \%$ de población no afiliada, no se notificaron casos de VIH en personas no afiliadas al sistema. Esto contrasta con los datos acopiados por la CAC del 2011 para el departamento, cuyo cálculo realizado por los investigadores permitió definir que 285 casos (8,8\%) de los infectados eran pobres no asegurados.

La planificación y ejecución de los programas obliga a la movilización de una gran cantidad de recursos, sin embargo, estos esfuerzos no se compadecen con las bajas coberturas de las actividades preventivas y la no inclusión de otros grupos de riesgo que pueden estar favoreciendo la alta incidencia hallada. En este sentido, se sugiere que se efectúe el seguimiento de la eficacia de las acciones, con enfoque diferencial y con criterios de equidad sanitaria, para dar respuesta a las necesidades en salud de cada grupo poblacional. En especial, se sugiere que los municipios fortalezcan sus acciones para aumentar las coberturas de prevención, en la población general, pero muy especialmente en poblaciones especiales de riesgo. También es importante reconocer los logros y tomar las medidas necesarias para afrontar los desafíos reportados por los coordinadores, para lograr el control efectivo de la epidemia.

Los resultados de este estudio muestran la imperiosa necesidad de formular una política municipal que acoja los lineamientos nacionales para la prevención de la epidemia, a partir de la condición de línea base de este estudio. En coherencia con las percepciones de los coordinadores de programa, se recomienda que los municipios introduzcan procesos formales de planificación, con asignación y gestión eficiente de los recursos (infraestructura, recursos técnicos, humanos y financieros), la participación de todos los actores responsables de la salud en el territorio, con el establecimiento de metas claras a corto y mediano plazo y determinación clara de las responsabilidades para cada uno de los actores involucrados.

También se requiere que la autoridad sanitaria proponga, vigile y controle, sobre la base de una evaluación permanente y sistemática de los logros propuestos. Estos logros no solo deben estar dirigidos a la disminución de la incidencia general y la garantía de una atención integral para los ya afectados, sino también aquellos referidos a la disminución del estigma, los cambios comportamentales relacionados con el uso del condón, el monitoreo de la disminución de la transmisión madre-hijo, así como la disminución en la problemática de grupos especiales. Estos elementos fueron sugeridos por los coordinadores de programa como logros, pero no se tienen bases empíricas que permitan corroborarlo en la práctica. 
La percepción de los programas de prevención por parte de los entes gubernamentales se encontraba divida. Aunque la apreciación de los coordinadores se dirigía a un enfoque preventivo (55\%), no existía coherencia entre los líderes de los programas y las EPS encargadas de la afiliación y atención de la población estudio. En comparación con otras investigaciones relacionadas con la evaluación de programas, se encontró que Rodríguez et al. presentan similitudes con respecto a los resultados, teniendo en cuenta que para el mejoramiento con respecto al desarrollo de los programas, se requiere talento humano interdisciplinario para la atención y prevención de la enfermedad, a pesar de que la otra investigación se centraba plenamente en los pacientes y no en los programas (20).

Aunque para la fecha del estudio la región tenía un $18 \%$ de población no afiliada, no se notificaron casos de VIH en personas no afiliadas al sistema. Esto contrasta con los datos acopiados por la CAC del 2011 para el departamento, cuyo cálculo realizado por los investigadores permitió definir que 285 casos $(8,8 \%)$ de los infectados eran pobres no asegurados.

Estas sugerencias están en sintonía con las recientes políticas expedidas en el Foro Latinoamericano y del Caribe sobre la atención del VIH, y la política internacional de Objetivos de Desarrollo Sostenible (ODS). En estas políticas se indica que los programas de prevención eficaces requieren la integración de los servicios de atención, que involucren a la comunidad, que se desarrollen con enfoque innovador y así entregar con eficiencia los servicios relacionados con el VIH (43). De conformidad con esta política, se propuso reducir en cinco años un 75\% de las nuevas infecciones para América Latina y el Caribe (41). Así mismo, la meta de ODS propone eliminar para el 2030 la patología (42). Es decir, que estos son algunos de los nuevos retos que los coordinadores de los programas para el VIH y demás actores del sistema deben enfrentar.

En conclusión, la región del suroeste tiene condiciones estructurales que pueden favorecer la epidemia, y sin embargo, la estructura (disponibilidad) y, los procesos que se llevan a cabo para el control de la epidemia resultan limitados. Las coberturas de las actividades son insuficientes y los resultados en salud, medidos en términos de incidencia, prevalencia y mortalidad son subóptimos.

\section{Limitaciones del estudio}

La calidad de los datos suministrados por los coordinadores de los programas está sujeta a la veracidad de los reportes y a la fiabilidad y completitud de los registros. En las bases de datos oficiales se observaron inconsistencias en el número de casos de VIH y la pérdida de datos se calculó en 10\%. Estas limitaciones no invalidan los resultados obtenidos.

\section{Agradecimientos}

Agradecemos, muy especialmente, a los coordinadores de programa participantes, a las secretarías de salud de salud que amablemente nos suministraron información, a la línea de investigación en Sistemas de Salud, al semillero Sissalud por el respaldo académico y administrativo y, en especial, a la gerente en sistemas de información Eliana López Holguín, por su apoyo en la formulación y ejecución del proyecto. El trabajo fue presentado en el IV Congreso Internacional de Sistemas de Salud y IV Conferencia Nacional de Salud Pública, organizado por la Pontificia Universidad Javeriana, Bogotá, entre el 3 y el 5 de octubre del 2016.

Este trabajo es producto de una investigación ejecutada en 2015 con recursos de la Estrategia de Sostenibilidad, CODI, de la Universidad de Antioquia. Por características especiales se entiende la caracterización de la población. Las poblaciones especiales consideradas fueron: adolescentes y adultos jóvenes entre 14 y 24 años, maternas, trabajadores sexuales, hombres que tienen sexo con hombres (HSH), niños menores de 14 años, desplazados, personas en situación de calle y población indígena. 


\section{Referencias bibliográficas}

1. Quintero Fleites EJ, Fe de la Mella Quintero S, Gómez López L. La promoción de la salud y su vínculo con la prevención primaria. Medicentro Electrónica. 2017;21(2):101-11.

2. Vignolo J, Vacarezza M, Álvarez C, Sosa A. Niveles de atención, de prevención y atención primaria de la salud. Archivos de Medicina Interna. 2011;33(1):7-11.

3. Congreso de Colombia. Ley 972 por la cual se adoptan normas para mejorar la atención por parte del Estado colombiano de la población que padece de enfermedades ruinosas o catastróficas, especialmente el VIH/Sida. Diario Oficial N.o 45.970. Bogotá, 2005.

4. Presidente de la Republica de Colombia. Decreto 1543. Por el cual se reglamenta el manejo de la infección por el Virus de Inmunodeficiencia Humana (VIH), Síndrome de la Inmunodeficiencia Adquirida (SIDA) y las otras Enfermedades de Transmisión Sexual (ETS). Diario Oficial N.o 43.062, Ministerio de Salud Publica. 1997.

5. Ministerio de Salud y Protección Social, Programa de Apoyo a la Reforma de Salud, Fundación para la Investigación y Desarrollo de la Salud. Modelo de gestión programática en VIH/SIDA. Colombia Guía para el manejo de VIH/SIDA. Basada en la evidencia Colombia [Internet]. 2006 [citado noviembre del 2015]. Disponible en: https://www.minsalud.gov.co/Documentos\%20y\%20Publicaciones/MODELO\%20DE\%20G ESTI\%C3\%93N\%20PARA\%20EL\%20MANEJO\%20DE\%20VIH\%20-SIDA.pdf

6. Ministerio de la Protección Social. Plan Decenal de Salud pública 2012-2021. Bogotá: Dimensión Sexualidad y Derechos Sexuales y Reproductivos; 2011.

7. Corte Constitucional. Sentencia T-760. Bogotá. 2008.

8. Górriz J, Otero A. Impacto socio sanitario de la enfermedad renal crónica avanzada. Rev Nefrología. 2008; 3(1):7-15.

9. Ministerio de la Protección Social. Decreto 3039. Por el cual se adopta el Plan Nacional de Salud Pública 2007-2010. Diario Oficial 46716. Bogotá. 2007

10. Sierra O, Lopera M, Chavez B, Montoya P. El Plan de Atención Básica en los municipios descentralizados de Colombia, 2001-2003. Rev Fac Nac Salud Pública. 2009;22(2):77-89.

11. Lopera M, Bula J. Impacto socioeconómico del VIH en las familias. Fenómeno olvidado en la política de atención integral Bogotá. En: Arrivillaga M, Useche B, editores. SIDA y sociedad crítica y desafíos sociales frente a la epidemia. Bogotá: Ediciones Aurora; 2011.p. 243-74.

12. Lopera M, Einarson T, Bula J. Out-of-pocket expenditures and coping strategies for people living with HIV. Aids Care. 2011;23(12):1602-8.

13. Lopera M, Martínez J, Einarson T. Acceso de las personas con VIH/SIDA al Sistema General de Seguridad Social en Salud y costos asociados desde la perspectiva individual y familiar. Bogotá, 2010. Rev Gerenc Polit Salud. 2010;10(20):81-96.

14. Ministerio de Salud y Protección Social. Análisis de Situación de Salud (ASIS). Bogotá: Dirección de Epidemiología y Demografía; 2015.

15. Ministerio de Salud y Protección Social. Boletín Epidemiológico, Situación del VIH/Sida. Bogotá: Ministerio de Salud; 2013.

16. Ministerio de Salud, Instituto Nacional de Salud. Mortalidad evitable en colombia para 1998-2011. Bogotá: Observatorio Nacional de Salud; 2014.

17. Secretaría Seccional de Salud y Protección Social. ITS (infecciones de transmisión sexual) 2000-2015 por municipio Antioquia. [Internet]. 2015 [citado 2017 oct. 8]. Disponible en: https://www.dssa.gov.co/index.php/estadisticas/eventos-en-salud-publica/item/73-its-infecciones-de-tran smision-sexual-2000-2012-por-municipio

18. Presidente de la República de Colombia. Decreto 0280. Objetivos de Desarrollo Sostenible (ODS). Agenda de Desarrollo Post-15 de la Organización de las Naciones Unidas. Bogotá: Presidencia de la Republica; 2015.

19. Ministerio de Salud y Protección Social. Resolución 1841 de 2013 por la cual se adopta el Plan Decenal de Salud Pública 2012-2021. Bogotá: El Ministerio; 2013. 
20. Congreso de la República. Ley 1438. Por medio de la cual se reforma el Sistema General de Seguridad Social en Salud y se dictan otras disposiciones. Diario Oficial 47957. Bogotá. 2011.

21. Congreso de la República de Colombia. Ley Estatutaria 1751, por medio de la cual se regula el derecho mundial a la salud y se dictan otras disposiciones. Diario Oficial 49427. Bogotá. 2008.

22. Deossa Restrepo G. Inequidades en salud relacionadas con el VIH. Perspect Nut Hum. 2009;11(3):93-07.

23. Rodríguez LA, Bautista JD. Evaluación de la calidad de los programas de atención integral del VIH/Sida en el municipio de Bucaramanga. Rev Univ Ind Santander, Salud. 2008; 40(3):215-21.

24. Scoppeta O. Discusión sobre evaluación de impacto de programas y proyectos sociales en salud pública. Univ Psychol Bogotá. 2006; 5(3):695-703.

25. Ureña J, Salazar S, Ojeda L, Mantilla T. Estudio de los costos en que incurren las entidades promotoras de salud en Colombia por la prevención y el tratamiento del VIH/SIDA. Cienc Tecnol Salud Vis Ocul. 2014; 12(1):51-63.

26. Estrada J. Modelos de prevenção na luta contra o HIV/AIDS. Acta Bioeth. 2006;12(1):91-100.

27. Donabedian A. Evaluating the quality of medical care. Milbank Mem Fund Q. 1966; 44(1):166-203.

28. Leavell H, Clark E. Preventive medicine for the doctor in his community. Tercera edición. Nueva York: McGrawHill; 1965.

29. Programa Conjunto de las Naciones Unidas. Manual sobe el VIH y los derechos humanos para las instituciones nacionales de derechos humanos. [Internet]. 2007 [citado 2015 jul. 14]. Disponible en: https://www.ohchr.org /Documents/Publications/HandbookHIV_NHRIs_sp.pdf

30. Congreso de Colombia. Ley 617. Por la cual se reforma parcialmente la Ley 136 de 1994, el Decreto extraordinario 1222 de 1886, se adiciona la Ley Orgánica de Presupuesto, el Decreto 1421 de 1993, se dictan otras normas tendientes a fortalecer la descentralizaciòn y se dictan normas para la racionalización del gasto público nacional. Diario Oficial 44188. Bogotá. 2000.

31. Ministerio de Salud y Protección Social. Lineamientos técnicos y metodológicos para el proceso territorial de generación de insumos concertados del Plan Decenal de Salud Pública 2012-2021. Bogotá: Ministerio de Salud; 2011.

32. Ministerio de la Protección Social. Modelo de gestión integral de servcios desalud sexual y reproductiva y prevención de la infección por VIH 2007. Bogotá: Ministerio de la Protección Social; 2007.

33. Council for International Oganizations of Medical Sciences, Organización Mundial de la Salud. Pautas éticas internacionales para la investigación biomédica en seres humanos. Programa Regional de Bioética OPS/OMS, Ginebra [Internet]. 2002 [citado 2015 dic. 2]. Disponible en: https://www.cioms.ch/publications/guidelines/ pautas_eticas_internacionales.htm

34. Council for International Oganizations of Medical Sciences, Organización Mundial de la Salud. Pautas internacionales para la evaluación ética de los estudios epidemiológicos. [Internet]. 2008 [citado 2015 dic. 2]. Disponible en: https://www.ms.gba.gov.ar/sitios/ccis/files/2012/08/PAUTASINTERNACIONALESEVAL UACIONETICAESTUDIOSEPIDEMIOLOGICOS1991.pdf

35. Asociación Médica Mundial. Declaración de Helsinki de la AMM - Principios éticos para las investigaciones médicas en seres humanos Helsinki, Filandia: Asociacion Medica Mundial; 2013.

36. Ministerio de Salud. Resolucion 8430. Por la cual se establecen las normas científicas, técnicas y administrativas para la investigación en salud. Instituto Nacional de Vigilancia de Medicamentos y Alimentos. Bogotá: Ministerio de Salud; 1993.

37. Gobernación de Antioquia. Departamento Administrativo de Planeación. Perfil de la Subregión del Suroeste. Antioquia. [Internet]. 2009 [citado 2015 jul. 18]. Disponible en: https://antioquia.gov.co/antioquia-v1/organ ismos/planeacion/descargas/perfiles/Perfil_subregional_Suroeste.pdf

38. Organización Mundial de la Salud. Estadísticas sanitarias mundiales. [Internet]. 2005 [citado 2016 abr. 2]. Disponible en: https://www.who.int/gho/publications/world_health_statistics/ES_WHS2005_Full.pdf?ua= 1 
39. Knobel H, Escobar I, Polo R. Recomendaciones GESIDA/SEFH/PNS para mejorar la adherencia al tratamiento antirretroviral en el año 2004. Enfermedades Infecciosas y Microbiología Clinica. 2004; 23(1):221-31.

40. Junta de Andalucía. Consejería de Salud y Bienestar Social. Equidad [Internet] [citado 2016 ene. 26]. Disponible en: https://www.calidadsaludandalucia.es/es/equidad.html

41. Secretaría Seccional de Antioquia y Protección social. Eventos de salud pública, Medellín. [Internet]. 2014 [citado 2015 jun. 6]. Disponible en: https://www.dssa.gov.co/index.php/estadisticas/eventos-en-salud-publica.

42. Varela M, Salazar I, Correa D. Adherencia al tratamiento en al infección por VIH/SIDA: consideraciones teóricas y metodológicas para su abordaje. Acta Colombiana de Psicología. 2008;11(2):101-13.

43. Foro Latinoamericano y del Caribe sobre el continuo de atención del VIH. Río de Janeiro. [Internet]. 2015 [citado 2015 dic. 16]. Disponible en: https://www.paho.org/col/index.php?option=com_content\&view=article\&id=2371:paises-de-america-la tina-y-el-caribe-se-comprometen-a-reducir-un-75-las-nuevas-infecciones-por-vih-en-adultos-y-jovenes-para-20 20\&Itemid $=551$

44. Asbford L. Cómo se ven afectadas las poblaciones por el VIH y el SIDA. [Internet]. 2006 [citado 2015 nov. 23]. Disponible en: https://www.prb.org/pdf06/howhivaidsaffectpop-sp.pdf

45. Guerrero H, Trujillo N. Inequidades en el acceso al sistema general de seguridad social en salud en Colombia. Universidad Pontificia Universidad Javeriana [Internet] [citado 2016 ene. 27]. Disponible en: https://repositor y.javeriana.edu.co/bitstream/10554/9015/1/GuerreroTorresHarrySalomon2013.pdf

\section{Licencia Creative Commons CC BY 4.0}

Para citar este artículo: Lopera Medina MM, Betancur Montoya T. Características de los programas de prevención primaria para el control del VIH en la región del suroeste antioqueño, 2014. Rev. Gerenc. Polit. Salud. 2018;17(35). https://doi.org/10.11144/Javeriana.rgps17-35.cppp 